\title{
Total Colonic Tubular Duplication Including Terminal Ileum and Appendix: A Rare Case
}

\author{
Mehmet Saraç, (1) Tugay Tartar, (1) Ünal Bakal, (1) Ahmet Kazez
}

Fırat University Faculty of Medicine, Department of Pediatric Surgery, Elazı̆̆, Turkey

\begin{abstract}
Total colonic duplications are rare. This case is reported here in order to discuss the treatment of tubular duplication of the entire colon, appendix and terminal ileum. A 2-year-old girl was admitted with complaints of vomiting, abdomen distension, and defecation difficulty. During laparotomy, a tubular duplication with separate mesentery which had no common intestinal wall was detected at the last $20 \mathrm{~cm}$ of the ileum. This duplication continued with a common wall and mesentery from the cecum to the sigmoid colon ending blindly. Resection anastomosis was performed for the completely separated tubular duplication at the terminal ileal segment. The remaining tubular duplication, continuing along the cecum, appendix and colon, could not be resected because of its common mesentery, vascular system, and wide common wall with the normal colon. Colotomy performed at the site of sigmoid colon allowed for the opening of the distal of the cyst to normal colon lumen. Gastrointestinal duplication cysts should be kept in mind in patients with chronic abdominal distension. Surgical treatment methods differ because of the different features and localizations of this tubular duplication.
\end{abstract}

Keywords: Total colonic tubular duplication, surgical treatment, child

\section{Introduction}

Gastrointestinal duplications (GID) are rare congenital anomalies that can be seen anywhere from the mouth to anus (1). It has been reported that their prevalence varies between $1 / 4,500$ to $1 / 10,000$. The colon is involved in only $13 \%$ of all duplications, and approximately 250 cases have been cited in the literature (2). $80 \%$ of patients have symptoms before 2 years of age (3). While $80 \%$ of GIDs have a spherical cystic structure and no connection with the gastrointestinal system, the remaining $20 \%$ are tubular in nature and they are associated with the lumen of the normal intestinal tract $(4,5)$. Total colonic tubular duplication also causes severe constipation and distension (6).

In this article, the treatment of a 2-year-old girl with a tubular intestinal duplication that included the terminal ileum, appendix, cecum, ascending colon, transverse colon, descending colon and sigmoid colon is presented in light of the literature.

\section{Case Report}

A 2-year-old girl was admitted with the complaints of vomiting, and abdomen distension. She had increasing abdominal distention which had started at the age of one. Her complaints of defecation difficulty and vomiting had begun in the two weeks prior to admission. Physical examination revealed a diffuse abdominal distension. There was an air-fluid level and widespread fecal accumulation was determined on direct abdominal radiography (Figure 1). No pathology was found in the abdominal ultrasonography. Contrast-enhanced abdominal computed tomography (CT) showed dilatation of the small intestine with the normal

\section{Address for Correspondence}

Tugay Tartar, Fırat University Faculty of Medicine, Department of Pediatric Surgery, Elazığ, Turkey Phone: +90 5066320100 E-mail: tugaytartar@gmail.com ORCID: orcid.org/0000-0002-7755-4736 Received: 18.01.2021 Accepted: 04.04.2021

${ }^{\circ}$ Copyright 2021 by Ege University Faculty of Medicine, Department of Pediatrics and Ege Children's Foundation The Journal of Pediatric Research, published by Galenos Publishing House. 


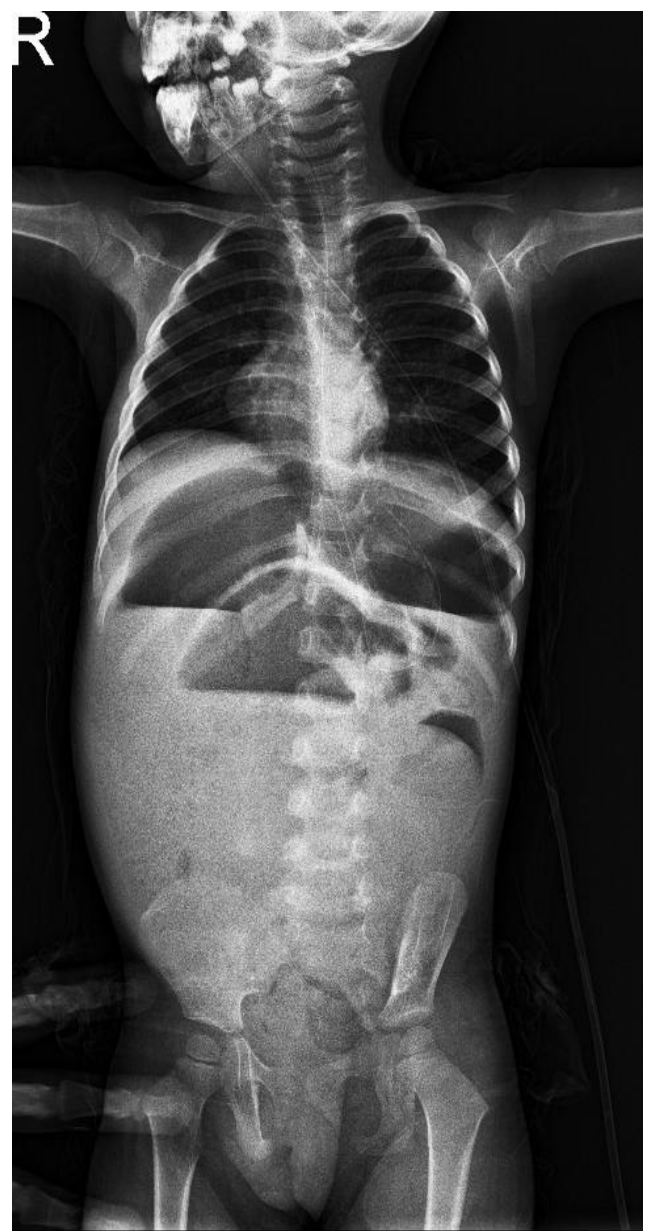

Figure 1. Air-fluid level and widespread fecal accumulation on the direct abdominal radiography

colon (Figure 2). The patient underwent laparotomy due to the absence of regression in the abdominal distension. Dilatation of the small intestines and accumulation of feces were seen. Furthermore, a tubular duplication with separate mesentery which had no common intestinal wall was detected at the last $20 \mathrm{~cm}$ of the ileum. This duplication continued along the cecum, appendix, ascending and descending colon and it ended blindly at the rectosigmoid region. The entire colonic tubular duplication, except for the distal ileum, had a common mesentery and a wide common intestinal wall on the antimesenteric surface of the colon (Figure 3). The duplicated lumen on the antimesenteric face was full of feces. It was observed that the initial section of the tubular duplication in the terminal ileum was connected to the actual lumen, and the continuing duplicated lumen ended blindly in the rectosigmoid region. Resection and ileo-ileal anastomosis were performed at the terminal ileal segment for the fully separated ileal duplication. The distal opening part of the duplication continuing with the cecum was closed. The remaining section of this duplication which

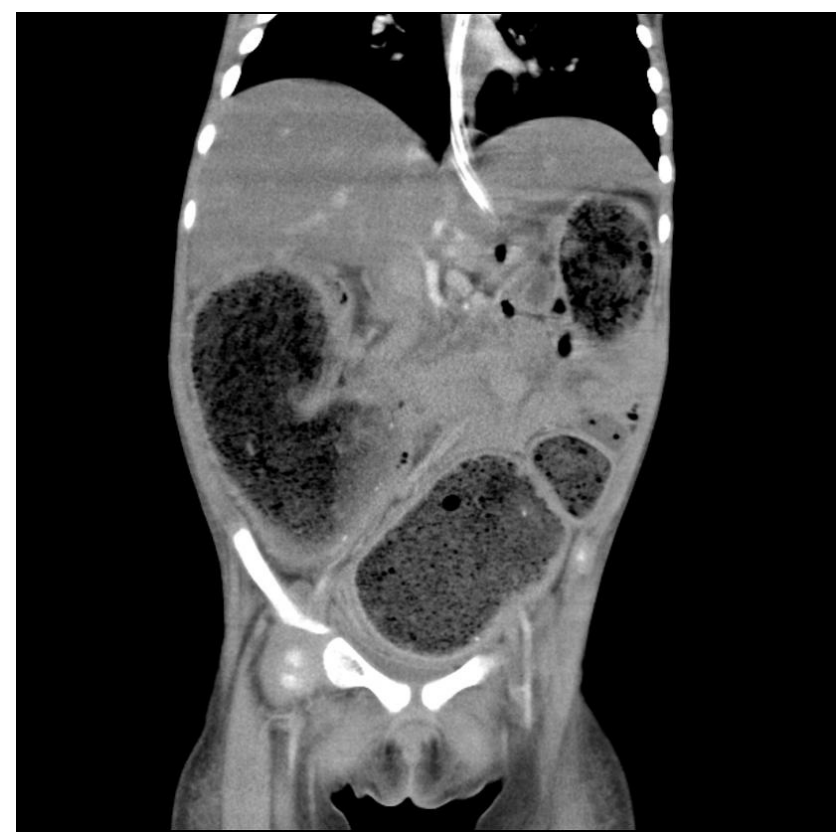

Figure 2. Contrast-enhanced abdominal computed tomography image

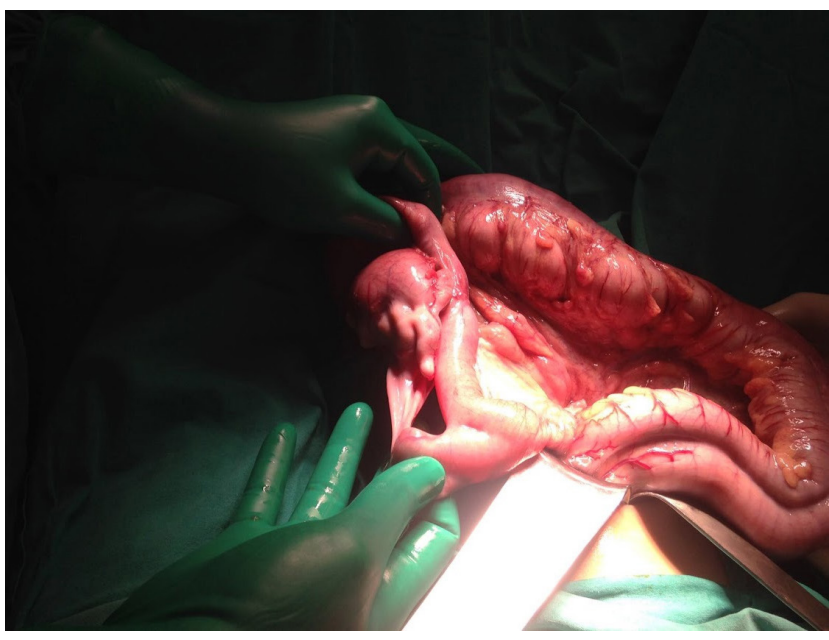

Figure 3. Preoperative image before surgery

continued along the entire colon could not be resected because of its common mesentery and wide common wall with the normal colon. Colotomy performed at the site of the sigmoid colon allowed for the opening of the distal of the tubular duplication to the normal colon lumen. Thus, contrary to the initial anatomy, the proximal opening of the duplex lumen was closed while the luminal opening was provided at the distal end (Figure 4). She did not require any further medical and/or surgical intervention after the operation. She produces stools regularly on a daily basis, and has been growing up without any complaint during 2 years of follow-up. Informed consent was received from the family before the preparation of this manuscript. 


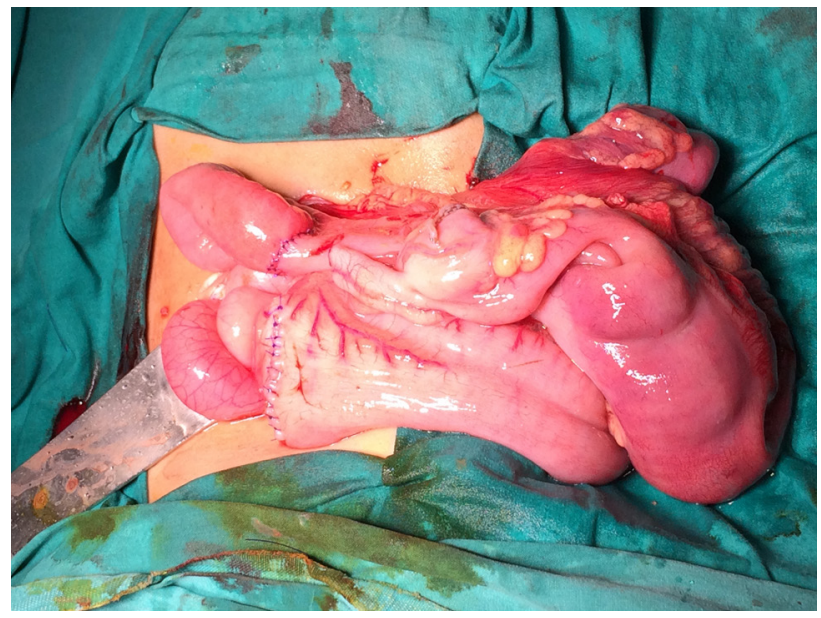

Figure 4. Preoperative image after surgery

\section{Discussion}

Although GID can be seen in any age group, they are mostly seen in children younger than two years of age $(3,5)$. The case presented here was in a two-year-old girl.

Colonic duplications are cystic or tubular in nature and constitute $13 \%$ of all duplication cysts (2). Tubular colonic duplication can show a function as two perineal anuses in the form of a double-barrelled blind ending, or they can function with a Y-shape as one or two lumen ends blindly such as imperforate anuses in distal (7). The colonic duplication in our case was tubular in nature and Y-shaped, ending blindly in the rectosigmoid junction.

GID contain well-developed smooth muscle in the wall and an epithelial lining resembling some part of the parent bowel (8). In our case, there was no common mesenteric, vascular structure or wall in the tubular duplication of the ileum, whereas the cecum, appendix, ascending colon, descending colon and rectosigmoid region had common mesentery, and a wide common wall with the normal intestine.

Tubular duplications are manifested by symptoms such as constipation, hematochezia, rectal prolapse, fistula, hemorrhoids and abscess according to their location $(4,6)$. Our case had abdominal distension which started at the age of one year and complaints of vomiting and defecation difficulty for two weeks prior to admission. In 15\% of patients, multiple duplication cysts can be seen (4). In our case, it was a single duplication, although tubular duplication continued in more than one area of the gastrointestinal system.

Standing direct abdomen radiography, barium imaging examinations, abdomen ultrasonography, CT and magnetic resonance imaging are used to identify duplication cysts
(8). Direct abdominal radiography, ultrasonography and CT were used in our case. However, the final diagnosis was made by diagnostic laparotomy.

Esophageal and gastric duplication cysts should be treated with cystectomy and other intestinal cysts should be treated either with cystectomy or resection anastomosis. Cystectomy is sufficient for duplication cysts without lumen connection with the enteric system. In cases where cystectomy is impossible, cystotomy and mucosectomy (Wreen procedure) is an option (9). Although malignant changes are reported in adults with duplication cysts (10), colorectal duplications are benign lesions. Therefore, radical surgical excision is not necessary. Surgical excision in asymptomatic cases is controversial. In addition, resection of the tubular duplicated colon may be impossible due to the presence of common mesentery in most cases (2). In our case, ileal tubular duplication was resected and colotomy performed at the sigmoid colon site in order to open the distal of the tubular colon to the normal colon lumen.

Complications related to surgery are post-operative bleeding, infection and bowel obstruction. However, in patients with total tubular duplication of the entire intestine, short bowel syndrome must be considered after such extensive surgery. Also, there are occasional reports of adenocarcinoma found in duplications, and they are not significantly different between communicating and non-communicating ones. The patient presented here will continue to receive follow-up to determine any possible malignancy.

In conclusion, GID should be kept in mind in patients with chronic abdominal distension and severe constipation. Surgical treatment methods differ due to the different characteristics and localizations of these duplications.

\section{Ethics}

Informed Consent: Informed consent was obtained from the parents of the patient for this study.

Peer-review: Externally peer-reviewed.

\section{Authorship Contributions}

Concept: M.S., T.T., Design: M.S., Ü.B., Data Collection or Processing: M.S, T.T., Ü.B., A.K., Analysis or Interpretation: M.S., T.T., A.K., Literature Search: M.S., Ü.B., Writing: M.S., T.T.

Conflict of Interest: No conflict of interest was declared by the authors.

Financial Disclosure: The authors declared that this study received no financial support. 


\section{References}

1. Kumar K, Dhull VS, Karunanithi S, et al. Synchronous thoracic and abdominal enteric duplication cysts: accurate detection with (99m)Tc-pertechnetate scintigraphy. Indian / Nucl Med 2015; 30:59-61.

2. Azahouani A, Hida M, Benhaddou H. Colonic duplication revealed by intestinal obstruction due to fecal impaction. Arch Pediatr 2015; 22:1284-7.

3. Kekez T, Augustin G, Hrstic I, et al. Colonic duplication in an adult who presented with chronic constipation attributed to hypothyroidism. World I Gastroenterol 2008; 14:644-6.

4. Khaleghnejad Tabari A, Mirshemirani A, Khaleghnejad Tabari N. Complete colonic duplication in children. Caspian I Intern Med 2012; 3:436-9.

5. Erginel B, Soysal FG, Ozbey $H$, et al. Enteric duplication cysts in children: a single-institution series with forty patients in twenty-six years. World I Surg 2017; 41:620-4.
6. Destro F, Pergola EL, Maestri L, Meroni M, Vella C, Riccipitetoni G. Total colonic tubular duplication causing severe constipation. J Pediatr Surg 2019; 48:101274.

7. Kaur N, Nagpal K, Sodhi P, Minocha VR. Hindgut duplication-case report and literature review. Pediatr Surg Int 2004; 20:6402.

8. Rattan KN, Bansal S, Dhamija A. Gastrointestinal duplication presenting as neonatal intestinal obstruction: an experience of 15 years at tertiary care centre. I Neonatal Surg 2017; 6:5.

9. Merrot $\mathrm{T}$, Anastasescu R, Pankevych $\mathrm{T}$, et al. Duodenal duplications. Clinical characteristics, embryological hypotheses, histological findings, treatment. Eur / Pediatr Surg 2006; 16:1823.

10. Inoue $\mathrm{Y}$, Nakamura $\mathrm{H}$. Adenocarcinoma arising in colonic duplication cysts with calcification: CT findings of two cases. Abdom Imaging 1998; 23:135-7. 